\title{
Brouwer's incomplete objects
}

\author{
Joop Niekus \\ ILLC, University of Amsterdam \\ j.m.niekus@uva.nl
}

\begin{abstract}
Brouwer's papers after 1945 are characterized by a technique, known as the method of the creating subject. It has been supposed that the method was radically new in his work, since Brouwer seems to introduce an idealized mathematician into his mathematical practice. A newly opened source, the unpublished text of a lecture of Brouwer from 1934, fully supports the conclusions of our analysis that:
\end{abstract}

-There is no idealized mathematician involved in the method.

-The method was not new at all.

-Brouwer uses an incomplete sequence, also known as choice sequence, in this method, which is special. The method does not take its place in the standard works on choice sequences.

\section{Introduction}

After a break of more than fifteen years Brouwer started to publish again in 1948. In the first paper of this new period, Brouwer 1948, he applied a technique which would become characteristic for his subsequent papers. In constructing a counterexample for classical logic he used a sequence of which the values depend on the activity of a creating subject. It has always been supposed that this method was for him a radically new approach. See for instance Heyting's comment on Kreisel's formalization of the method:

It is true that Brouwer in his lecture introduced an entirely new idea for which the subject is of essential importance. As I said, I feel that it is still questionable whether it is possible and whether it is good to introduce this idea in mathematics. ${ }^{1}$

Kreisel's formalization was elaborated by Troelstra. Brouwer's basic term creating subject was changed in creative subject and interpreted as the idealized

\footnotetext{
${ }^{1}$ See Lakatos 1967 , p. 173. The lecture Heyting refers to is Consciousness, Philosophy and Mathematics, Brouwer 1975, p. 480-495.
} 
mathematician, all its activity divided into $\omega$ discrete stages. The resulting theory of the creative subject, TCS for short, is strong enough to derive most of Brouwer's creating subject arguments and it can be added consistently to the intuitionistic logic. But the TCS cannot give a satisfactory reconstruction of Brouwer's argument, and worse, there are serious conceptual problems. From seemingly plausible assumptions about an idealized mathematician Troelstra derived a paradox which could not be resolved in a satisfactory manner. The theory became the standard theory, and thus we shall refer to it; but the method remained controversial, see Troelstra's own words:

It should be stressed at this point that talking about the 'idealized mathematician' in no way commits us to adopt the speculative features of Brouwer's theory of the 'creative subject' (the idealized mathematician); that is, first the possibility of explicit reference to the course of mathematical activity of the idealized mathematician in constructions by the idealized mathematician, and secondly the division of all mathematical activity into $\omega$ discrete stages.

This quote is from the introduction of his monograph Choice Sequences (Troelstra 1977), which is the standard text on that subject. Brouwer introduced choice sequences in 1918 to solve his foundational problems. They are sequences not completely determined by a law; Brouwer called them incomplete. The notion of choice sequence is far from completely clarified, as we shall see in this paper.

No connection has been made between the standard theories of choice sequences and the TCS; the two theories have been developed completely separately. In the intuitionistic handbooks a considerable amount of space is reserved for the theories on choice sequences, while the method of the creating subject is treated in the last few pages. See for example Dummett 1977 and van Dalen and Troelstra 1988. In the latter reference the authors remark, after discussing the paradox and their unsatisfactory solutions of it, that

In this connection the solution proposed by Niekus 1987 deserves

further investigation.

In our Niekus 1987 we discussed the example from Brouwer 1948. We pointed out that the change from creating subject to creative subject was misleading. According to Brouwer's view mathematics is a creation of the human mind and by using the expression creating subject Brouwer only made explicit his idealistic position; it can be replaced by we or $I$. Interpreted in this way an idealized mathematician is not needed at all for the reconstruction, a simple principle for reasoning about the future is enough. The theory resulting from our analysis does not suffer the shortcomings of the standard theory. The criticism quoted above applies to the standard reconstruction, but not to our way of interpreting Brouwer's argument. 
At the end of our paper we remarked that Brouwer's supposedly new method is nothing but the use of a choice sequence. It is not the introduction of an idealized mathematician, but the systematic application of individual choice sequence, that makes the method under discussion special. Individual choice sequences used by Brouwer have not been studied in the research on choice sequences, except for one possible example, viz. the sequence Brouwer uses in his proof of the negative continuity theorem in Brouwer 192\%. But this example is vague and deviates essentially from all his other uses of choice sequences, see section 5 .

As we stated above the method has for many years been supposed to be a new one for Brouwer, notwithstanding his remark in Brouwer 1948 that he had used the method in his lectures from 1927 onwards. This remark was a puzzle; see for example Troelstra 1982 p. 479:

A reconstruction which would be based on the 'solipsistic explanation' of the creative subject seems us to be undoubtedly anachronistic; though Brouwer 1948 states he had been using such arguments since 1927 , he certainly did not use them in his publications before 1948, [...].

Or see van Dalen and Troelstra 1988, p. 842:

The theory of the idealized mathematician or 'creative subject' [...]; its origin is to be found in Brouwer 1948 (according to Brouwer, the idea dates back to 1927).

But in 1991 the text of a Brouwer lecture from 1927 was published. The editor van Dalen notices a choice sequence in this text, and he connects it, cautiously, with the method of the creating subject, see Brouwer (1991) p. 1314. This conforms completely to our interpretation, since this example is the same as the one in 1948, except that Brouwer uses we instead of creating subject. A similar choice sequence occurs already in Brouwer 1930, but this time Brouwer uses I instead of creating subject, see Niekus 2005 and section 6 of this paper.

More outspoken is van Dalen in van Dalen 19996 (p. 394), were he writes about the 1927 example

The method was later called the method of the creating subject.

This statement shows that van Dalen has switched from creative subject to creating subject and that he accepts our interpretation of the term creating subject, since Brouwer uses we instead of creating subject in this example. But he does not fully accept the consequences of this interpretation. He remains faithful to the standard theory. For his results 
in van Dalen 1999a he uses Kripke's Scheme, a principle derived from the standard theory, but not valid according to our reconstruction, see section 4 below. Also other authors in recent publications, e.g. Dummett 2000 and van Atten 2004, still adhere to the standard theory and do not mention the connection with earlier work. And in van Atten 200\%, a philosophical study towards the origin of choice sequences, creating subject sequences do not occur. The only example in it of a choice sequence used by Brouwer, is the deviating one in his proof of negative continuity we mentioned above.

In this somewhat unclear situation a new source became available which gives in our opinion definite evidence for our position. It is the text of a lecture Brouwer gave in 1934 (Brouwer 1934). The text seems very important for understanding choice sequences, for there is no place in the work of Brouwer where he is so explicit about their nature. It shows without doubt that Brouwer started to use individual choice sequences in the late twenties, and that he elaborated their use in his papers after 1945. But above all, the text fully supports the conception of an incomplete object underlying our reconstruction of Brouwer's creating subject arguments.

The material of the paper is arranged as follows. We start in section 2 with a description of the standard theory for the reconstruction of the supposedly new method, the TCS. In section 3 we discuss what we think are two of its shortcomings. First, the theory does not explain Brouwer's argument satisfactorily. Second, the notion of the idealized mathematician is very problematic, if not paradoxical.

In section 4 we present our reconstruction. We do not use an idealized mathematician, only a principle of reasoning about the future that we think is obvious. We end this section by summarizing the conception of an incomplete sequence that arises from our reconstruction. After the introduction of incomplete sequences in section 5 , and the demonstration of its first examples in section 6, we shall see in section 7 that this conception is fully supported by the rich source Brouwer 1934.

\section{The theory of the creative subject}

In his first paper after 1945 Brouwer defines a real number for which he proves that it is different from 0 , but not apart from 0 . This definition, from Brouwer 1948, runs as follows:

Let $\alpha$ be a mathematical assertion that cannot be tested, i.e. for which no method is known to prove either its absurdity or 
the absurdity of its absurdity. Then the creating subject can, in connection with this assertion $\alpha$, create an infinitely proceeding sequence $a_{1}, a_{2}, a_{3}, \ldots$ according to the following direction: As long as, in the course of choosing the $a_{n}$, the creating subject has experienced neither the truth, nor the absurdity of $\alpha, a_{n}$ is chosen equal to 0 . However, as soon as between the choice of $a_{r-1}$ and $a_{r}$ the creating subject has obtained a proof of the truth of $\alpha, a_{r}$ as well as $a_{r+v}$ for every natural number $v$ is chosen equal to $2^{-r}$. And as soon as between the choice of $a_{s-1}$ and $a_{s}$ the creating subject has experienced the absurdity of $\alpha, a_{s}$, as well as $a_{s+v}$ for every natural number $v$ is chosen equal to $-(2)^{-s}$. This infinitely proceeding sequence $a_{1}, a_{2}, a_{3}, \ldots$ is positively convergent, so it defines a real number $\rho$ (Brouwer 1975, p. 478).

Although Brouwer stated in the introduction of the paper that he had used this example in his lectures already from 1927 onwards, it was supposed that this way of defining a sequence was new for him. Brouwer seemingly used the activity of a subject to define a sequence. In this manner it was interpreted in the reconstruction of Kreisel 1967, which was further elaborated in Myhill 1968, and in particular in Troelstra 1969. In the resulting theory the expression creating subject was changed to creative subject and this creative subject was taken to be an idealized mathematician, for short $I M$, all of its mathematical activities supposedly divided into a discrete sequence of $\omega$ stages. The key notion in this theory of the creative subject, for short $T C S$, is: the creative subject has evidence for $\varphi$ at stage $n$, formally expressed by

$$
I_{n} \varphi
$$

Analyzing the properties of an idealized mathematician then leads to the acceptance of the following axioms ( $n$ and $m$ are natural numbers, $\varphi$ can be any mathematical assertion):

(1) $I_{n} \varphi \vee \neg I_{n} \varphi$,

(2) $I_{n} \varphi \rightarrow I_{n+m} \varphi$,

(3) $\varphi \rightarrow \exists n I_{n} \varphi$

(4) $\exists n I_{n} \varphi \rightarrow \varphi$.

We will not discuss these axioms, but we shall look at their consequences. We just mention the following. They can be added consistently to the intuitionistic logic, as is evident from the construction of a model 
by van Dalen (van Dalen 1978). Furthermore, the theory has become the standard in intuitionistic research; it has recently been applied by authors on the subject, e.g. Dummett (Dummett 2000) and van Atten (van Atten 2004), for the reconstruction of Brouwer's supposedly new method.

With the TCS Brouwer's result can be obtained as follows. Let $A$ be an undecided proposition, i.e. neither $A$ nor $\neg A$ is known. We define an infinite sequence $a_{1}, a_{2}, a_{3} \ldots$, by

$$
\begin{aligned}
& a_{n}=0 \leftrightarrow\left(\neg I_{n} A \wedge \neg I_{n} \neg A\right), \\
& a_{n}=2^{-m} \leftrightarrow\left(m<n \wedge \neg I_{m} A \wedge I_{m+1} A\right), \\
& a_{n}=-2^{-m} \leftrightarrow\left(m<n \wedge \neg I_{m} \neg A \wedge I_{m+1} \neg A\right) .
\end{aligned}
$$

The sequence $a_{1}, a_{2}, a_{3}, \ldots$ defines a real number, say $\rho$. If for this real number $\rho>0$ were to hold, than $A$ would hold as well because of

$$
\begin{aligned}
& \rho>0 \rightarrow \exists n I_{n} A, \quad \text { by the definition of } \rho, \text { and } \\
& \exists n I_{n} A \rightarrow A, \quad \text { by (4). }
\end{aligned}
$$

Since $A$ is undecided, $A$ does not hold, so neither does $\rho>0$. Analogously, $\rho<0$ does not hold, because then $\neg A$ would follow. So $\rho$ is not apart from 0 . But since

$$
\begin{aligned}
& \rho=0 \rightarrow \neg \rho>0 \\
& \neg \rho>0 \rightarrow \neg \exists n I_{n} A, \quad \text { by the definition of } \rho, \text { and } \\
& \neg \exists n I_{n} A \rightarrow \neg A, \quad \text { by the contraposition of (3) }
\end{aligned}
$$

$\rho=0 \rightarrow \neg A$ holds. Analogously we have $\rho=0 \rightarrow \neg \neg A$, and consequently $\rho=0 \rightarrow(\neg A \wedge \neg \neg A)$. So $\rho \neq 0$ does hold; apartness and equality are not equivalent, concluding the derivation of Brouwer's result.

For any mathematical assertion $A$ we can define in a way similar similar to the above $\left(a(n)=0 \leftrightarrow \neg I_{n} A\right)$ and $\left(a(n)=1 \leftrightarrow I_{n} A\right)$. This results in the axiom scheme which is known as Kripke's Scheme:

$$
\text { KS } \exists a(\forall n(a(n)=0 \vee a(n)=1) \wedge \exists n(a(n) \neq 0 \leftrightarrow A)) .
$$


$K S$ is often accepted as a reasonable principle. It is strong enough to derive most of Brouwer's counterexamples and for that reason it is sometimes added to the intuitionistic logic instead of the axioms of the $T C S$. Its advantage is that it does not explicitly refer to an idealized mathematician.

As stated in the introduction, we do not accept the standard reconstruction embodied in the TCS, and neither do we see any basis in Brouwer's work for accepting the principle $K S$. We will explain this in the next section.

\section{The theory of the creative subject-II}

A striking feature of the reconstruction of section 2 is that the untestedness in Brouwer's definition of $\rho$ is not used and not needed; undecidability seems to be sufficient. Inspecting Brouwer's original proof shows that his argument for $\rho \neq 0$ is the same as in the reconstruction, but that his argument for $\rho>0$ does not hold is different. It runs as follows:

If for this real number $\rho$ the relation $\rho>0$ were to hold, then $\rho<0$ would be impossible, so it would be certain $\alpha$ could never be proved to be absurd, so the absurdity of the absurdity of $\alpha$ would be known, so $\alpha$ would be tested, which it is not. Thus the relation $\rho>0$ does not hold (Brouwer 1975, p. 478-479).

This reasoning to conclude testedness from $\rho>0$ can be expressed in language of the TCS by

$$
\begin{aligned}
& \rho>0 \rightarrow \neg \rho<0, \\
& \neg \rho<0 \rightarrow \neg \exists n I_{n} \neg A, \quad \text { by the definition of } \rho, \text { and } \\
& \neg \exists n I_{n} \neg A \rightarrow \neg \neg A, \quad \text { by the contraposition of (3). }
\end{aligned}
$$

As we may observe, Brouwer's reasoning is more complicated than the reconstruction of the TCS in section 2. The reason is that Brouwer does not use (4) here. Neither does he do so in his proof for $\rho<0$, which is analogous, nor in his proof for $\rho \neq 0$, which is the same as in the $T C S$, see section 2 . The use of (4) would simplify his argument, and he would not need to resort to an untested proposition, but could have used an undecided one. It seems to us that he does not want to use (4).

But the reason that the TCS is widely considered to be controversial is another one. To demonstrate the difficulties involved we exhibit a paradox discovered by Troelstra (Troelstra 1969, pp. 105-107). 
Suppose that the CS proves his results one by one. By sufficiently narrowing the stages this amounts to one new result by the CS at each stage. Let $A_{0}, A_{1}, A_{2}, \ldots$ be the list of new results. We now define a predicate $L(\alpha)$ such that $L(\alpha)$ holds if and only if $\alpha$ is a lawlike sequence. We define a sequence $\beta$ such that

$$
\begin{aligned}
& \beta(n)=\alpha(n)+1 \leftrightarrow A_{n}=L(\alpha) \text { for some } \alpha \\
& \beta(n)=0 \text { otherwise. }
\end{aligned}
$$

Now Troelstra argues that intuitively $\beta$ is lawlike, since it is determined by some fixed recipe, so $L(\beta)$ holds. Because of (4) we have $\exists n I_{n} L(\beta)$, so for some $n_{0}, I_{n_{0}} L(\beta)$ holds. But then $\beta\left(n_{0}\right)=\beta\left(n_{0}\right)+1$, which is a contradiction.

Troelstra discusses two ways out. The first is to drop the condition of one new result at each stage; the second is to bring onto the stages a type structure of levels of selfreflection. He judges neither of them satisfactory, and he concludes that 'the attempts to formalize the theory of the $I M$ as envisaged by Brouwer cannot be said to be satisfactory examples of "informal" rigour' (van Dalen and Troelstra 1988, p. 846).

An argument for taking $\beta$ to be lawlike may be that in the TCS the stages seem to have a definite description, expressed by (1). But in the intuitionistic interpretation, for a disjunction to hold we need a proof of one of the disjunctive parts. In the case of $\beta$ this seems not evident to us.

Let us return to Brouwer's original use of creating subject, let us interpret it as ourselves and let the stages cover our future. We can define $\beta$ as above. Then its values depend on our future results. We have no way to determine these values, other than going in time to these stages, which are not specified at all. We think decidability is questionable, and we do not want to call this sequence lawlike. In section 6 and 7 below we shall show that Brouwer uses creating subject sequences as examples of sequences not completely determined by a law.

Our conclusion of this section is that (1) and (4) are problematic. In the next section we shall look for principles for the reconstruction of Brouwer's argument, in our interpretation as indicated above. We shall see that our analogues of these two principles turn out to be not valid. 


\section{A new reconstruction}

If we interpret the expression creating subject as ourselves, the sequence $a_{1}, a_{2}, a_{3}, \ldots$ from the definition in section 2 is a sequence that we may construct. We then start by choosing $a_{1}=0$ and we let $a_{n}=a_{n+1}$ with one exception. If we find a proof of $A$ between the choice of $a_{r-1}$ and $a_{r}$ we choose $a_{r+v}=2^{-r}$ for every $v$; and if we find a proof of not $-A$ between the choice of $a_{r-1}$ and $a_{r}$ we choose $a_{r+v}=-2^{-r}$ for every $v$.

The values of the sequence now depend on our future mathematical results. We want intuitionistically valid principles to reason about them. For our basic term we shall use $G$ instead of $I$, to stress the point that our interpretation is radically different. The $G$ is used in temporal logic to express "it is going to be the case that", and we shall use it similarly.

We imagine our future to be covered by a discrete sequence of $\omega$ stages, starting with the present stage as stage 0 , and we define for a mathematical assertion $\varphi$

$$
G_{n} \varphi
$$

as: at the $n$-th stage from now we shall have a proof of $\varphi$. The introduction of this term enables us to refine the notion of proof.

In intuitionism stating $\varphi$ means stating the possession of a proof of $\varphi$. We now demand of such a proof that it can be carried out here and now, i.e. all information for the proof is available at the present stage. If future information is involved we use $G_{n} \varphi$. A proof of $G_{n} \varphi$ may depend on information coming free before stage $n$. Of course $G_{n} \varphi$ may also hold because we have a proof for $\varphi$ already now; we suppose a proof to remain valid. So we have (for any $\varphi, n$ and $m$ ):

(5) $\varphi \rightarrow \exists n G_{n} \varphi$.

Of course we also have for all $n$ and $m$

(6) $G_{n} \varphi \rightarrow G_{n+m} \varphi$.

But we can not accept the analogues of (1) and (4). That

(7) $\exists n G_{n} \varphi \rightarrow \varphi$

is not valid can we see as follows. Let $B$ be an undecided proposition. We define a sequence $b_{1}, b_{2}, b_{3} \ldots$ as follows. We choose $b_{n}=0$ until we have found a proof for $B \vee \neg B$, after which we keep $b_{n}=1$. 
Take $\varphi$ to be $\left(b_{n_{0}}=0 \vee b_{n_{0}}=1\right)$. We do not have $\left(b_{n_{0}}=0 \vee b_{n_{0}}=1\right)$ for any $n_{0}$, because this would mean that we would have one of the disjuncts, and so we would already know now, whether we shall have a proof of $B \vee \neg B$ at the time of the choice of $b_{n_{0}}$. On the other hand, we do have $G_{n_{0}+1}\left(b_{n_{0}}=0 \vee b_{n_{0}}=1\right)$, i.e. we do have $G_{n_{0}+1} \varphi$, so $(7)$ does not hold. In a similar way it follows that

(8) $G_{n} \varphi \vee \neg G_{n} \varphi$

is not valid. Let the sequence $b_{1}, b_{2}, b_{3} \ldots$ be defined as above and take $\varphi$ to be $b_{n_{0}}=1$. If (8) were to hold, we would know already now whether we shall have a proof of $B \vee \neg B$ at the time of the choice of $b_{n_{0}}$, which is of course not generally true: (8) does not hold either.

So, as extra principles above intuitionistic logic, we only have (5) and (6). But this is enough, because an inspection of Brouwer's proof, see above, shows that we only need the contraposition of (5). Thus, the untestedness of $\alpha$ becomes crucial. ${ }^{2}$

Since we do not have (7), we cannot derive $K S$, which is in accordance with Brouwer's practice. Although there are one or more instances of $K S$ for specific cases in the work of Brouwer, he always carefully avoided its use as a general principle for an unspecified formula, see e.g. section 3. ${ }^{3}$ We found support for our reconstruction in the following quote. It is the translation by Heyting of a handwritten note in Dutch found with his 1949 proof of a strong counterexample; see Brouwer 1975, p. 603-604:

Further distinctions in connection with the excluded middle.

$\bar{a}$ will mean: $a$ is non-contradictory.

$\underline{a}$ will mean: $a$ is contradictory

$b$ implies a will mean: from now on I have an algorithm which enables me to derive $a$ from $b$.

The principle of testability can assert:

either: from now on either $\bar{a}$ or $\underline{a}$ holds, notation: $\mid a$.

or: from a certain moment in future on either $\bar{a}$ or $\underline{a}$ will hold, notation $a \mid$.

\footnotetext{
${ }^{2}$ Our basic term $G_{n} \varphi$ has a very natural interpretation in a certain kind of Kripke model, see Niekus 198\%. For these models (5) and (6) are valid formulas for every $\varphi$. But (8) is not valid, and (7) is only valid for negative formulas.

${ }^{3}$ There is an instance of $K S$ in Brouwer's work, from the last year in which he published, see Brouwer 1975, p. 525, eleventh line from below. Whether there are arguments for this specific instance of $K S$ remains an interesting question.
} 
Then $a \mid$ is non-contradictory, but $\mid a$ need not to be non-contradictory. For instance, let $p$ be a point of the continuum in course of development, whose continuation is free at this moment, but may be restricted at any moment in the future; then ( $p$ is rational) is non-contradictory, but $\mid$ ( $p$ is rational) is contradictory, for the complete freedom which exists at this moment makes it impossible to be sure that the rationality of $p$ is contradictory, but also to be sure that it is contradictory that the rationality of $p$ is contradictory.

In struggling with his new notion of tensed objects he comes up with an explicit distinction, which is the same as we make. For his $a \mid$ and $\mid a$ are the same as our $\neg \neg a \vee \neg a$ and $G_{n}(\neg \neg a \vee \neg a)$. At the end of this note Brouwer expresses doubts about introducing $\mid a$ as a mathematical notion, without further argument. But we focus here on the logical distinction. That Brouwer, given the distinction, would accept (7) is of course out of question: stating that $\neg \neg a \vee \neg a$ is contradictory and that $G_{n}(\neg \neg a \vee \neg a)$ is not, refutes (7) in a very strong way. We conclude there is no base for $K S$ in Brouwer's creating subject arguments.

We interpreted the expression creating subject as we, and anybody else can interpret it as himself. Brouwer's definition is a description of a construction, as any intuitionistic definition. But the construction is not completely determined. The values of the sequence under consideration depend on the mathematical experience of the maker of the sequence, the creating subject. But the activity of the creating subject is not used in the proof. The reasoning is done on the basis of the incomplete description only, before the construction has started.

In 1918 Brouwer introduced incomplete sequences, also known as choice sequences, to solve his foundational problems i.e. to attain the power of the continuum from the discrete. We claim that Brouwer is using an incomplete sequence in the example discussed above. It is not the introduction of an idealized mathematician that makes the creating subject arguments special, but the application of individual incomplete objects. In the next sections we will seek in the work of Brouwer support for our position.

\section{The definition of a spread}

In intuitionism mathematics is a creation of the human individual. It consists of mental constructions by the individual only. Prime material for these constructions is the sequence of the natural numbers $N$, which 
has its origin in "our perception of a move of time" (Brouwer 1981, p. 4). From the natural numbers the integers $Z$ and rational numbers $Q$ can be constructed. Brouwer was this far already in his thesis in 1907 (Brouwer (1975), p. 11-98). But at that time $N$ and $Q$ were actual infinite sets in Brouwer's conception, and he did not have a satisfactory method to introduce the real numbers. By 1918 he had solved his foundational problems and he had drawn the full consequences of his constructivist point of view. The sequence of natural numbers was given by its first element and a law to construct every next one. So $N$, and thereby $Q$ and $Z$, were potentially infinite sets. And the real numbers were also introduced by a method to construct them: the notion of a spread. It is this notion that made the intuitionistic reconstruction of mathematics possible. We give here a slightly modified version.

The definition of a spread is founded on a countable sequence $A$ of mathematical objects already constructed. $A$ may for example consist of natural numbers, rational numbers or intervals of rational numbers. A spread is a law that regulates the construction of infinitely proceeding sequences, their terms chosen more or less freely from $A$. The law says, in constructing a sequence, whether a choice from $A$ is admissible as first element, and whether it is admissible as next in an already constructed initial segment. After each admitted choice there is at least one admissible successor. A sequence constructed according to a spread law is called an element of the spread. Such an element is generally not completely representable.

In his originally German text Brouwer used for spread the word Menge, which is German for set. This may be misleading, because a spread is not defined by its elements, but provides a way to construct them. So, quantifying over elements of a spread is quantifying over sequences one can construct. This construction does not have to be fixed beforehand completely. The terms of an element are chosen one by one, and within the limitations of the spread law, these choices are free. But at any moment of the construction this freedom can be limited further.

If a sequence is completely determined from the first term onward, we call the sequence lawlike. Brouwer names them sharp, or fertig, which is German for completed. If a sequence is not completely determined we call it, following Brouwer, incomplete. In previous papers (Niekus 1987 and Niekus 2005) we used for incomplete sequences the term choice sequences, in the tradition of Heyting and Troelstra. In this meaning it is also used below. But in the literature choice sequence sometimes is used for an arbitrary element of a spread, lawlike or not.

The fact that an arbitrary element of a spread is constructed term 
by term, with no other restriction than the spread law, leads to the continuity principle: if a property holds for an arbitrary element of a spread, this must be evident on the basis of a finite initial segment, and then it holds as a matter of fact for every element with the same initial segment. Brouwer applies this principle to prove the existence of uncountable powers as follows (Brouwer 1975, p. 160).

Let $C$ be the spread with as founding sequence the natural numbers $N$, and each choice admissible. Its elements are sequences of natural numbers. If to each element of this spread a natural number is assigned by a function $f$, the assignment must be done on the basis of an initial segment of each element, and any element of $C$ sharing the same initial segment, will be assigned the same number. So $f$ cannot be 1-1. Conversely, a 1-1 function from $N$ to $C$ is easily indicated. Conclusion: $C$ has a larger power than $N$.

The continuity principle is not valid classically: the function $f$ assigning a 0 to sequences of natural numbers with all the terms even, and 1 otherwise, is classically a perfect definition. In Intuitionism it is not.

These general principles of a continuum with choice sequences were the main interest in the research on choice sequences. The standard text on the subject is the monograph Troelstra 197\%. It contains a huge number of technical results on formal systems of certain classes of choice sequences. For these formal systems Troelstra proves elimination theorems: a sentence with quantification over choice sequences can be translated into an equivalent sentence without parameters for choice sequences. From these technical results he draws the conclusion that all choice sequences are eliminable. 'They have no mathematical relevance', he states in his recent lectures, 'their interest is philosophical' (Troelstra 2001, p. 227).

There are no instances of individual choice sequences in Troelstra 1977. But there is one in Troelstra 1982, a study on the origin and development of choice sequences in the work of Brouwer. It is a sequence Brouwer used in his proof of the negative continuity theorem, preceding his famous and stronger continuity theorem (Brouwer 1927). This proof of the negative continuity theorem is so vague that it allows many reconstructions. Troelstra discusses three possible ones, and there are others, see e.g. Posy 1976 and Martino 1985, all different. It is also the only Brouwerian choice sequence discussed in van Atten 2007 (p. 108-109), a recent study on the phenomenology of choice sequences. But in no way this sequence can be called characteristic for Brouwer's use of individual choice sequences, for the following reason. 
When he proved the theorem again, along the same lines but this time more explicit, he clearly uses a lawlike sequence, see Brouwer 1981, p. 81. At that moment Brouwer was fully exploiting individual choice sequences in the method of the creating subject, a use he started in 1927. We shall show that in the next two sections, after the necessary definitions.

\section{$6 \quad$ Incomplete objects}

The real numbers can be introduced by a spread, with as founding sequence an enumeration of the rational numbers. Any rational number $q$ is admissible as first choice. A rational number $q$ is admissible as next in an admitted initial segment $q_{1}, q_{2}, \ldots, q_{n}$ if $\left|q-q_{n}\right|<2^{-n}$. So, the elements of this spread are convergent sequences of rational numbers. Two elements $\left(a_{n}\right)$ and $\left(b_{n}\right)$ are coincident if their termwise difference converges to 0, i.e. if $\forall k \exists n \forall m>n\left(\left|a_{m+n}-b_{n+m}\right|<2^{-k}\right)$. Real numbers are introduced as equivalence classes of this relation.

All handling of real numbers is done via their generating sequences. For example, for the real numbers $a$ and $b$, generated by $\left(a_{n}\right)$ and $\left(b_{n}\right)$, $a<b$ holds if $\exists n \exists k \forall m\left(\left(b_{n+m}-a_{n+m}\right)>2^{-k}\right)$ holds.

This is by no means the only way. Brouwer had a preference to introduce the real numbers by a spread with as founding sequence an enumeration of the $\lambda^{(n)}$-intervals; a $\lambda^{(n)}$-interval is an interval of rational numbers of the form $\left[a \cdot 2^{-n},(a+2) \cdot 2^{-n}\right]$, with $a$ an integer. An element of this spread is a sequence with as $n$-th term a $\lambda^{(n)}$ - interval which is contained in its predecessor. Two sequences of $\lambda^{(n)}$-intervals, Brouwer calls them points, are now coincident, when each interval of one of the sequences, has an interval in common with every interval of the other sequence. Real numbers, point cores, are again the equivalence classes of the coincidence relation. For real numbers $a$ and $b$ we now define $a<b$ if an interval of the generating sequence of $a$ is lying to the left of an interval of the generating sequence of $b$.

There is no essential difference between these methods of introducing real numbers. They result in the same continuum.

For real numbers Brouwer made the following distinction. The real numbers generated by lawlike sequences form the reduced continuum; all real numbers together, generated by lawlike or incomplete elements, form the (full) continuum. He mentions this distinction already in 1919 (Brouwer 1975, p. 235) but he started to work with it from 1927 onwards. 
In that year Brouwer gave lectures in Berlin; the text of these lectures is in Brouwer 1991.

Relevant for our purpose in this text is Brouwer's study of the notion of order. He shows that $<$ is not a complete order on the reduced continuum, i.e. he shows that $a=0 \vee a<0 \vee a>0$ does not hold generally for the real numbers generated by lawlike sequences. In doing this he uses a technique already applied by him in 1908 (Brouwer 1975, p. 108). ${ }^{4}$

Further, we denote with $K_{1}$ the smallest natural number $n$ with the property that the $n$-th up to the $(n+9)$-th digit in the decimal expansion of $\pi$ form the sequence 0123456789 , and we define as follows a point $r$ of the reduced continuum: the $n$-th $\lambda$-interval $\lambda_{n}$ is a $\lambda^{(n-1)}$-interval centered around 0 , as long as $n<K_{1}$; however, for $n \geq K_{1} \lambda_{n}$ is a $\lambda^{(n-1)}$-interval centered around $(-2)^{-K_{1}}$. The point core of the reduced continuum generated by $r$ is neither $=0$, nor $<0$ nor $>0$, as long as the existence of $K_{1}$ neither has been proved nor has been proved to be absurd. So until one of these discoveries has taken place the reduced continuum is not completely ordered (Brouwer 1991, p. 31-32).

Note the role of time in this argument. Neither $r<0$ nor $r>0$ did hold for Brouwer then and there, because he could not give a specific natural number $n_{0}$ such that $K_{1}=n_{0}$. Recently it has been discovered that $K_{1}$ exists and that it is even, so $r>0$ holds now (Borwein 1998). For the full continuum he proves that the relation $<$ is not an order at all, i.e. $a \neq 0 \rightarrow(a>0 \vee a<0)$ does not hold for every $a$. This proof is new:

Therefore we consider a mathematical entity or species $S$, a property $E$, and we define as follows the point $s$ of the continuum: the $n$-th $\lambda$-interval $\lambda_{n}$ is a $\lambda^{(n-1)}$-interval centered around 0 , as long as neither the validity nor the absurdity of $E$ for $S$ is known, but it is a $\lambda^{(n)}$-interval centered around $2^{-m}\left(-2^{-m}\right)$, if $n \geq m$ and between the choice of the $(m-1)$-th and the $m$-th interval a proof of the validity (absurdity) of $E$ for $S$ has been found. The point core belonging to $s$ is $\neq 0$, but as long as neither the absurdity, nor the absurdity of the absurdity of $E$ for $S$ is known, neither $>0$ nor $<0$. Until one of these discoveries has taken place, the continuum cannot be ordered (Brouwer 1991, p. 31-32).

Brouwer proves here a stronger statement for the full continuum than he does for the reduced continuum: if a relation on a space is not an

\footnotetext{
${ }^{4}$ The citations in this section are translations by the author from the German original.
} 
order, it cannot be a complete order. Therefore, the sequence generating $s$ in the citation above must be an incomplete sequence.

As we remarked in section 2, Brouwer mentioned in the introduction of his 1948 example that he had used it in his lectures from 1927 onwards. We think it cannot be otherwise than that he referred to the example above; there is no other candidate in Brouwer 1991, nor is there in the other texts of lectures we shall discuss below. Note that Brouwer uses we in his definition, in conformity with our interpretation of the creating subject.

This example has not played a role in the discussion about the creating subject arguments: it was not published until 1991. But a year after Berlin Brouwer gave similar examples in a lecture in Vienna, and this text was published in 1930, see Brouwer 1930.

He had generalized the technique based on the expansion of $\pi$ as used in the first Berlin example, by introducing the notion of a fleeing property for natural numbers. It satisfies the following conditions: for each natural number it is decidable whether it possesses $f$ or not, no natural number possessing $f$ is known, and the assumption of the existence of a number possessing $f$ is not known to be contradictory. The critical number $\lambda_{f}$ of a fleeing property $f$ is the smallest natural number possessing $f$.

Brouwer's standard example of a fleeing property is being the smallest $k$, the $k$-th up to the $k+9$-th digit in $\pi$ 's expansion of which form the sequence $0,1,2, \ldots, 9$, used in the first cited Berlin example above. The real number defined over there is an example of a dual pendular number (our translation of the German duale Pendelzahl). As we mentioned in the previous section, for Brouwer's standard example the critical number has become known, so for us this property is not fleeing anymore.

In Brouwer 1930 Brouwer examines the continuum on seven properties, all valid classically, but not intuitionistically. Whenever it is possible, he uses a lawlike sequence, as in the following example. With 0 instead of $1 / 2$ it is the same as our first cited Berlin example.

That the continuum (and also the reduced continuum) is not discrete follows from e.g. the fact that the number $1 / 2+p_{f}$, where $p_{f}$ is the dual pendular number of the fleeing property $f$, is neither equal to $1 / 2$, nor apart from $1 / 2$. (Brouwer 1975 , p. 435).

But if necessary he uses an incomplete sequence

That $<$ is not an order on the continuum is demonstrated by the real number $p$, generated by the sequence $\left(c_{n}\right)$, its terms chosen 
such that $c_{1}=0$ and $c_{v}=c_{v+1}$ with only the following exception. Whenever I find the critical number of some particular fleeing property $f$, I choose the next $c_{v}$ equal to $-2^{-v}$, and when I find a proof this critical number does not exist, I choose $c_{v}$ equal to $2^{-v}$. This number $p$ is unequal to 0 , but nevertheless it is not apart from zero (Brouwer 1975, p. 435-436).

Notwithstanding the superficial similarity, this sequence $\left(c_{n}\right)$ is completely different from the sequence $\left(b_{n}\right)$ used in the Berlin example to show that the reduced continuum was not completely ordered. There is no 1-1 connection between the values of $\left(c_{n}\right)$ and the digits of the expansion of $\pi$ as in the case of $\left(b_{n}\right) ;\left(c_{n}\right)$ is used for a property of the full continuum, so it is an incomplete sequence. In the next section we shall cite Brouwer about the difference between the two kinds of sequences.

The resemblance with the 1948 example is less than in the case of the Berlin example, because Brouwer does not mention untestedness here. Whether it is the same depends on the question whether a fleeing property can be tested or not, which is not obvious from the definition Brouwer gives here. We think that clearly Brouwer had the intention to give the same example, and that he supposes untestedness, for the following reason. If the non-existence of a number possessing $f$ is known to be contradictory, one of the possibilities in the definition above would be excluded beforehand. Furthermore, Brouwer gives in Brouwer 1948 his standard example of a fleeing property also as an example of an untested proposition.

Let us return to the starting point of our historical review, which was finding evidence for our reconstruction of the 1948 creating subject argument. As we have seen in this section, the method of the CS was not new for Brouwer in 1948. We have also concluded that Brouwer applies in this method incomplete elements of a spread.

The citations in the next section, which are from our richest source, confirm these conclusions. But above all, these citations support our conception of an incomplete sequence.

\section{$7 \quad$ The Geneva Lectures}

Brouwer stopped publishing after 1930 but he did not stop lecturing. Brouwer 1934 is the text of his Geneva lectures of 1934. In no way this manuscript was made fit for publication. It is full of crossing outs and improvements. But there is no other place in the work of Brouwer where 
he spends so many words on the special character of incomplete objects. Furthermore, this text is the link between Brouwer's creating subject arguments after 1948 and the first choice sequences of the late twenties we discussed above.

The text contains no new results. After the introduction of the real numbers with $\lambda$-intervals and the definition of order, he wants to show that the natural $<$ is not an order on the continuum. Therefore he defines a real number by giving a description of a construction: ${ }^{5}$

The $n$-th interval $\lambda^{(n-1)}$ is of length $2 /(n-1)$ and centered around 0 . This is how one starts. But at the same time one works on a difficult problem, to know whether the property $E$ for a species $S$ is true, for example Fermat's problem. If for that problem a solution has been found between the $(n-1)$-th and the $n$-th choice, the choice of the intervals will be different.

As we pointed out in the example of Brouwer (1930), if $E$ could be tested, one of the possibilities in the definition would be excluded. We remark that Brouwer gives in Brouwer (1948) also Fermat's problem as an example of a proposition that cannot be tested.

If the property is true for the species $S$, then the $v$-th interval will be for $v \geq n$ the interval $\lambda^{(v)}$ centered around $2^{-n}$. The next interval will be placed according to this law, within its predecessor with the same center. If, on the other side, one finds that the property $E$ is absurd for the species $S$, than the intervals will be centered around $-2^{-n}$.

Brouwer proves, just as he will do again in 1948, that the defined number cannot be equal to 0 :

This point $s$ is defined completely correctly. The point is different from 0 , because if it was equal to 0 , then the possibility to continue the sequence around $2^{-n}$ would be excluded. So the supposition that one day a proof of $E$ for $S$ would be found, would be absurd and the supposition that one day one finds a proof of the absurdity for $S$ would be absurd too. The truth and the absurdity of that property would both be absurd and that is impossible.

Next he argues that the point cannot have a positive distance from 0 :

\footnotetext{
${ }^{5}$ Brouwer 1934 consists of six parts, probably corresponding to six lectures. All quotatios of this section are from the second part, pp. 22-26, translated by the author from the French original.
} 
We have a point which is different from 0 , but it is neither positive nor negative, because if it was the one or the other, the problem in question would be solved.

But the following text has been struck out:

We have a point that is different from 0 , but at the same time $[\ldots]$ (if we define that point by $s$ ), neither the relation $s<0$, nor the relation $>$ than 0 holds, because, if the relation $s<0$ would hold, one would have to exclude the first variant, that is, one would have solved the problem positively, which is not the case, and if the other relation would hold, one should have to exclude the second variant, that is to say that one would have solved the problem negatively.

We do not know the reason why he scrapped the proof. But that he did not trust the proof seems unthinkable, because he used the same proof later, in 1948. Further, there can be no misunderstanding about the fact that he is using an incomplete sequence here:

We show the same for the reduced continuum. The point above is not a sharp point, because the construction is not completely determined, but depends on the intelligence of the constructor relative to the posed problem.

Constructor is our translation of the French constructeur. As it seems, Brouwer would opt later for creating subject. That $>$ is not an order on the reduced continuum (actually Brouwer only shows that it not a complete order) is proved in the familiar way. Let $K_{1}$ be defined as in the Berlin example. One starts with choosing as $\lambda_{n}$ a $\lambda^{(n)}$-interval centered around 0 for $n<K_{1}$, and continues by choosing a $\lambda^{(n)}$-interval centered around $(-2)^{-K_{1}}$ for $n \geq K_{1}$. About the difference between this point $r$ and $s$, the incomplete sequence above, Brouwer remarks:

When one hundred different persons are constructing the number $r$, one is always certain that any interval chosen by one of these persons is always covered, at least partly, by every interval chosen by one of the others. That is different for $s$. If I would give the definition of $s$ to one hundred different persons, who are all going to work in a different room, it is possible that one of these one hundred persons at one time will choose an interval not covered by an interval chosen by one of the others.

As we observe here, there is no idealized mathematician involved in these incomplete sequences. They are given by a description of a construction, 
their terms are made to depend on the future mathematical experience of the one who constructs them, which can be anyone. The future activity of this subject does not play a role, the reasoning about an incomplete sequence is done before the construction has started, on the basis of the incomplete description only.

\section{Acknowledgement}

The research for this article has been made possible by support of the Teacher in Research programme of the Netherlands Organization of Scientific Research (NWO). I thank NWO for giving this support. I am much indebted to Mark van Atten for an exchange of views. I thank Theo Janssen for his patience, his comments and his support. I thank Dick de Jongh for his careful reading and stimulating supervision. Finally, I thank the ILLC (Institute for Logic, Language and Computation of the University of Amsterdam) for its hospitality and support.

\section{References}

van Atten, M., 2004. On Brouwer, Wadsworth Philosophers Series, London: Thomson Learning.

van Atten, M., 2007. Brouwer meets Husserl, Synthese Library 335, Dordrecht, The Netherlands: Springer.

Borwein, J. M., 1998. 'Brouwer-Heyting sequences converge', The Mathematical Intelligencer, 20, 14-15.

Brouwer, L. E. J., 1927. 'Über Definitionsbereiche van Funktionen', Mathematische annalen, 97, 60-75, also Brouwer 1975, 390-405.

Brouwer, L. E. J., 1930. 'Die Struktur des Kontinuums', Vienna: Gottlieb Gistel. Also Brouwer 1975, 429-440.

Brouwer, L. E. J., 1934. 'Geneva lectures 1934', Brouwer Archive BMS 44 - unpublished.

Brouwer, L. E. J., 1948. 'Essentieel negatieve eigenschappen', Proceedings KoninklijkeNederlandse Akademie van Wetenschappen, 51, 963964, english translation: Essentially negative properties,Indagationes Math., 10: 322-323. Brouwer 1975, 478-479. 
Brouwer, L. E. J., 1975. Collected Works, vol. I, North Holland Publishing Company, editor A. Heyting.

Brouwer, L. E. J., 1981. Brouwer's Cambridge lectures on intuitionism, Cambridge University Press, editor D. van Dalen.

Brouwer, L. E. J., 1991. Intuitionismus, B.I. Wissenschaftverlag, editor D. van Dalen.

van Dalen, D., 1978. 'An interpretation of intuitionistic analysis', Annals of mathematical logic, 13, 1-43.

van Dalen, D., 1999a. 'From Brouwerian counterexamples to the creating subject', Studia logica, 62, 305-314.

van Dalen, D., 1999b. Mystic, geometer and intuitionist - The life of L. E. J. Brouwer, Clarendon Press Oxford.

van Dalen, D. and Troelstra, A. S. (eds.), 1982. The L. E. J. Brouwer centenary symposium, North Holland Publishing Company.

van Dalen, D. and Troelstra, A. S., 1988. Constructivity in mathematics, vol. I and II, Amsterdam: North Holland Publishing Company.

Dummett, M., 1977. Elements of intuitionism, Oxford Logic Guides 39, Oxford: Clarendon Press, first edn.

Dummett, M., 2000. Elements of intuitionism, second edition, Oxford Logic Guides 39, Oxford: Clarendon Press, second edn.

Kreisel, G., 1967. Informal rigour and completeness proofs, in Lakatos 1967, 138-186.

Lakatos, I. (ed.), 1967. Problems in the philosophy of mathematics, vol. I, North-Holland Publishing Company, proceedings of the International Colloquium in the Philosophy of Science, London, 1965.

Martino, E., 1985. 'On the Brouwerian concept of negative continuity', Journal of Philosophical Logic, 14, 379-398.

Myhill, J., 1968. 'Formal systems of intuitionistic analysis I', In: Logic, methodology and philosophy of science III, editors B. van Rootselaer and J. Staal, North Holland Publishing Company, Amsterdam.

Niekus, J. M., 1987. 'The method of the creative subject', Proceedings of the Koninklijke Akademie van Wetenschappen te Amsterdam, Series A(4), 431-443. 
Niekus, J. M., 2005. 'Individual choice sequences in the work of L. E. J. Brouwer', Philosophia Scientiae, Cahier spécial 5, 217-233.

Posy, C., 1976. 'Varieties of indeterminacy in the theory of general choice sequences', Journal of Philosophical Logic, 5, 431-443.

Troelstra, A. S., 1969. Principles of intuitionism, vol. 95 of Springer Lecture Notes, Springer Verlag.

Troelstra, A. S., 1977. Choice sequences, Oxford Logic Guides, Clarendon Press.

Troelstra, A. S., 1982. 'On the origin and development of Brouwer's concept of choice sequences', In van Dalen and Troelstra 1982.

Troelstra, A. S., 2001. 'Honderd jaar keuzerijen', Verslag van de gewone vergadering van de afdeling natuurkunde, 24-09-2001, volume 110, nr.6, 223-227, lecture for the ordinary meeting of the section physics of the Royal Dutch Academy of Science, 24-09-2001. 